\section{Bivalent HPV vaccine safety depending on subtypes of juvenile idiopathic arthritis}

We read with great interest the article by Heijstek $e a^{1}{ }^{1}$ regarding the safety of the bivalent human papillomavirus (HPV) vaccine in patients with juvenile idiopathic arthritis (JIA). Their prospective controlled observational cohort study of 68 patients revealed no differences in adverse events between female JIA patients and healthy girls. The authors concluded that the vaccine is well tolerated in JIA patients. Most reports regarding the safety of the HPV vaccine, including premarket cohort studies $^{2-4}$ and postlicensing safety monitoring surveys, ${ }^{5}$ suggest no apparent causal relationship between the vaccine and serious adverse events (SAEs) in young girls. Now the focus has moved to vaccine safety in patients with illnesses such as immunological disorders. ${ }^{6}$ Heijstek et $a l^{1}$ did report longer durations of arthralgia, fatigue and headache after vaccination in JIA patients compared with healthy controls. In addition, SAEs occurred more often in JIA patients, suggesting there could be definite differences in immunological reactions and symptomatic involvement between JIA patients and healthy subjects. This is consistent with our recent experience of a 15 -year-old JIA patient who developed severe pain and walking disability after injection of the bivalent HPV vaccine. She had had presentations of intermittent buttock pain and lumbago 2 years before vaccination. A few days after the first dose, she had persistent pain with joint swelling, which spread to multiple sites after further doses of the vaccine. She was diagnosed with enthesitis type JIA, confirmed by power-Doppler ultrasonography, and had bone erosion at the entheses. In this case, it was unclear whether vaccination uncovered underlying disease or aggravated the disease, which had been overlooked, but the clinical phenotype was obvious polyenthesitis. Polyenthesitis is a chronic, non-traumatic inflammatory disorder. Most children with polyenthesitis have enthesitis type JIA, which is associated with a high prevalence of inflammatory back pain related to sacroiliitis, HLA-B27, uveitis, inflammatory bowel disease, ankylosing spondylitis and reactive arthritis in the past, present or in the family. ${ }^{7}$ Heijstek et $a l^{1}$ mentioned that five of 68 JIA patients had SAEs: three were diagnosed with inflammatory bowel disease within 1 year after vaccination, one patient was admitted with transient lower back pain and one patient had uveitis. These clinical features are rarely seen in polyarticular or oligoarticular type JIA, but are common in enthesitis type JIA. This, together with our experience, may suggest that polyenthesitis is a key pathogenesis for inducing pain and adverse reactions after HPV vaccination.

However, it is unclear whether a patient with enthesitis type JIA has a greater risk of an SAE after HPV vaccination, because only one enthesitis case was recruited in the study in this article.
Indeed, the authors did not describe the characteristics of patients who had SAEs after vaccination. It is also unknown whether four cases with HLA-B27 developed SAEs or not. To clarify the risk factors in SAEs after HPV vaccination, the next cohort study should be performed in cases with polyenthesitis or enthesitis type JIA. It is important to clarify the risk of SAEs and dispel unfounded suspicions regarding the HPV vaccine.

\section{Shinji Akioka}

Correspondence to Dr Shinji Akioka, Department of Pediatrics, Kyoto Prefectural University of Medicine, Kyoto 6028566, Japan; sakioka@koto.kpu-m.ac.jp

\section{Competing interests None.}

Provenance and peer review Not commissioned; externally peer reviewed.

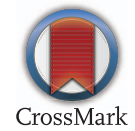

To cite Akioka S. Ann Rheum Dis 2014;73:e75.

Received 6 August 2014

Accepted 10 August 2014

Published Online First 27 August 2014

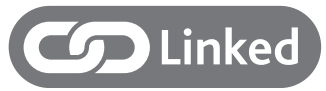

http://dx.doi.org/10.1136/annrheumdis-2014-206446

Ann Rheum Dis 2014;73:e75. doi:10.1136/annrheumdis-2014-206426

\section{REFERENCES}

1 Heijstek MW, Scherpenisse M, Groot N, et al. Immunogenicity and safety of the bivalent HPV vaccine in female patients with juvenile idiopathic arthritis: a prospective controlled observational cohort study. Ann Rheum Dis 2014;73:1500-7.

2 Harper DM, Franco EL, Wheeler C, et al. Efficacy of a bivalent L1 virus -like particle vaccine in prevention of infection with human papillomavirus types 16 and 18 in young women: a randomised controlled trial. Lancet 2004;364:1757-65.

3 Paavonen J, Naud P, Salmeron J, et al. Efficacy of human papillomavirus (HPV)-16/18 AS04-adjuvanted vaccine against cervical infection and precancer caused by oncogenic HPV types (PATRICIA): final analysis of a double-blind, randomised study in young women. Lancet 2009;374:301-14.

4 Lu B, Kumar A, Castellsague $X$, et al. Efficacy and safety of prophylactic vaccines against cervical HPV infection and diseases among women: a systematic review \& meta-analysis. BMC Infect Dis 2011;11:13.

5 Stokley S, Jeyarajah J, Yankey D, et al. Human papillomavirus vaccination coverage among adolescents, 2007-2013, and postlicensure vaccine safety monitoring, 20062014-United States. MMWR Morb Mortal Wkly Rep 2014;63:620-4.

6 Pellegrino P, Carnovale C, Pozzi M, et al. On the relationship between human papilloma virus vaccine and autoimmune diseases. Autoimmun Rev 2014; 13:736-41.

7 Petty RE, Southwood TR, Manners P, et al. International League of Associations for Rheumatology classification of juvenile idiopathic arthritis: second revision, Edmonton, 2001. J Rheumatol 2004;31:390-2. 